\title{
Tensile behaviour of geopolymer-based materials under medium and high strain rates
}

\author{
Costantino Menna ${ }^{1, \text { a }}$, Domenico Asprone ${ }^{1}$, Daniele Forni ${ }^{2}$, Giuseppina Roviello $^{3}$, Laura Ricciotti ${ }^{3}$, Claudio Ferone $^{3}$, \\ Anna Bozza ${ }^{1}$, Andrea Prota ${ }^{1}$, and Ezio Cadoni ${ }^{2}$ \\ ${ }^{1}$ Department of Structures for Engineering and Architecture, University of Naples Federico II, 80125 Naples, Italy \\ ${ }^{2}$ DynaMat Laboratory, University of Applied Sciences of Southern Switzerland, 6952 Canobbio, Switzerland \\ ${ }^{3}$ Dipartimento di Ingegneria, University of Naples Parthenope, Centro Direzionale Napoli, 80143 Naples, Italy
}

\begin{abstract}
Geopolymers are a promising class of inorganic materials typically obtained from an alluminosilicate source and an alkaline solution, and characterized by an amorphous 3-D framework structure. These materials are particularly attractive for the construction industry due to mechanical and environmental advantages they exhibit compared to conventional systems. Indeed, geopolymer-based concretes represent a challenge for the large scale uses of such a binder material and many research studies currently focus on this topic. However, the behaviour of geopolymers under high dynamic loads is rarely investigated, even though it is of a fundamental concern for the integrity/vulnerability assessment under extreme dynamic events. The present study aims to investigate the effect of high dynamic loading conditions on the tensile behaviour of different geopolymer formulations. The dynamic tests were performed under different strain rates by using a Hydro-pneumatic machine and a modified Hopkinson bar at the DynaMat laboratory of the University of Applied Sciences of Southern Switzerland. The results are processed in terms of stress-strain relationships and strength dynamic increase factor at different strain-rate levels. The dynamic increase factor was also compared with CEB recommendations. The experimental outcomes can be used to assess the constitutive laws of geopolymers under dynamic load conditions and implemented into analytical models.
\end{abstract}

\section{Introduction}

Geopolymers, also known as "alkali-activated cements" or "alkali-activated aluminosilicates", represent a new class of high performance inorganic materials characterized by a three dimensional, $\mathrm{CaO}$-free, silico-aluminate chemical structure [1]. They can be produced by mixing reactive aluminosilicate materials such as metakaolin [2,3], industrial [4] or natural waste products [5], and strongly alkaline solutions such as $\mathrm{NaOH}$ or $\mathrm{KOH}$, resulting in a rigid geopolymeric network.

These materials are widely studied since they show interesting mechanical properties, thermal stability, freezethaw, chemical and fire resistance, long term durability and environmental advantages; for these reasons, their applications cover many fields.

First of all, geopolymer materials represent a "greener" alternative of conventional Ordinary Portland Cement (OPC) in new Sustainable Buildings due to several environmental advantages, such as a lower $\mathrm{CO}_{2}$ footprint and low production temperature. Geopolymer technology also entails inexpensive and eco-friendly synthetic procedures, encompassing clays and other natural silicoaluminates as well as industrial wastes like coal fly ash and sediments [6]. Moreover, at elevated temperatures, geopolymer concretes exhibit less potential to suffer from spalling in comparison with OPC concrete.

Recently, geopolymer-based hybrid composite systems have been also investigated in order to overcome some

${ }^{\text {a }}$ Corresponding author: costantino.menna@unina.it issues linked to the brittle behaviour, the unknown longterm durability, and rheological behaviour of geopolymers that usually restrict the possible number of "in-situ" applications as structural materials. Among them, a great interest has been devoted to polymeric fillers due to their low density, chemical stability and easy processing. It was reported that hybrid composite materials based on geopolymers containing an organic epoxy resin content (up to $25 \%$ wt) can be effectively achieved by carrying out a direct polymerization in situ, simultaneously to the geopolymeric polycondensation reaction $[7,8]$.

As far as geopolymer and geopolymer hybrid composite mechanical properties are concerned, most of the experimental works have been conducted within the quasi-static regime with the aim of determining the compressive strength, compressive Young's modulus, Poisson's ratio, splitting tensile strength, and fracture energy. Moreover, while strain rate effect on OPC concrete has been extensively studied, only a few studies have been conducted on the dynamic properties of geopolymer-based concretes, mainly focused on damping and compressive behaviour.

In particular, the damping behaviour of geopolymer pastes was investigated by Pan et al. [9], showing comparable performances with OPC pastes. Khandelwal et al. [10] investigated the strain rate effect on the Young's modulus, Poisson's ratio and compressive strength of low-calcium fly-ash-based geopolymer mortars. It was found that these properties substantially increase in a logarithmic manner as the strain rate increases. Recently, Feng et al. [11] investigated the effect of strain rate on

This is an Open Access article distributed under the terms of the Creative Commons Attribution License 4.0, which permits unrestricted use, distribution, and reproduction in any medium, provided the original work is properly cited. 
the compressive behaviour of geopolymer concrete and mortar by means of a split Hopkinson pressure bar in the range of $10^{-6}-370 \mathrm{~s}^{-1}$ of strain rate. They found that the dynamic increase factor for compressive strength and the corresponding critical strain increased with the increasing strain rates and $\mathrm{CEB}$ recommendations underestimated this factor in the case of geopolymer concrete.

Tensile behavior under high strain rates has not been investigated yet, as far as the authors know, in available literature. However dynamic tensile behavior is of large interest for understanding the fracture development and the energy absorbing capabilities of these kinds of materials, when subjected to impulsive loads and impacts.

In the present work, the tensile behavior of a metakaolin based geopolymer and two hybrid geopolymer composites is investigated. Direct tensile and splitting tests are performed using an Hydro-pneumatic machine and a modified Hopkinson bars, both installed at the DynaMat laboratory of the University of Applied Sciences of Southern Switzerland.

\section{Materials and experimental methods}

\subsection{Geopolymer-based materials}

Three different geopolymer-based materials are investigated and tested under dynamic conditions: a metakaolin based geopolymer (neat geopolymer, hereafter referred as GEO), an hybrid organic resin-geopolymer composite (GEO-Epoxy) and a hybrid inorganic resin-geopolymer composite (GEO-SIL). GEO samples were obtained starting form an alkaline activating solution prepared by dissolving solid sodium hydroxide into a sodium silicate solution with a composition expressed as $\mathrm{Na}_{2} \mathrm{O} 1.4 \mathrm{SiO}_{2}$ $10.5 \mathrm{H}_{2} \mathrm{O}$ [12]. Then, metakaolin was incorporated into the activating solution with a liquid to solid ratio of $1.4: 1$ by weight, and mixed by a mechanical mixer for $10 \mathrm{~min}$ at $800 \mathrm{rpm}$. The composition of the whole geopolymeric system can be expressed as $\mathrm{Al}_{2} \mathrm{O}_{3} \cdot 3.5 \mathrm{SiO}_{2} \cdot 1.0 \mathrm{Na}_{2} \mathrm{O}$. $10.5 \mathrm{H}_{2} \mathrm{O}$, assuming that the geopolymerization occurred at $100 \%$.

Hybrid-organic (Geo-Epoxy) and inorganic (Geo-SIL) samples were prepared by incorporating a commercial epoxy and siloxanic resin, according to the $10 \%$ of weight content, into the freshly prepared geopolymeric suspension under mechanical stirring, when the polycondensation reactions of the components were already started but far to be completed $[8,13]$.

Afterwards, a set of samples was poured in cylindrical moulds and cured at $60{ }^{\circ} \mathrm{C}$ for $48 \mathrm{~h}$ and then kept in $>$ $95 \%$ relative humidity conditions at room temperature for further 5 days. Finally, the specimens were kept further 21 days in air at room temperature. For all the samples, the evaporation of water was prevented by sealing the top of the moulds with a plastic film during the curing stage.

\subsection{Experimental setup}

The tensile dynamic behaviour of the investigated geopolymer-based samples was investigated by means of direct tensile and splitting tests. Both testing configurations were performed on $20 \mathrm{~mm}$ high cylindrical specimens with

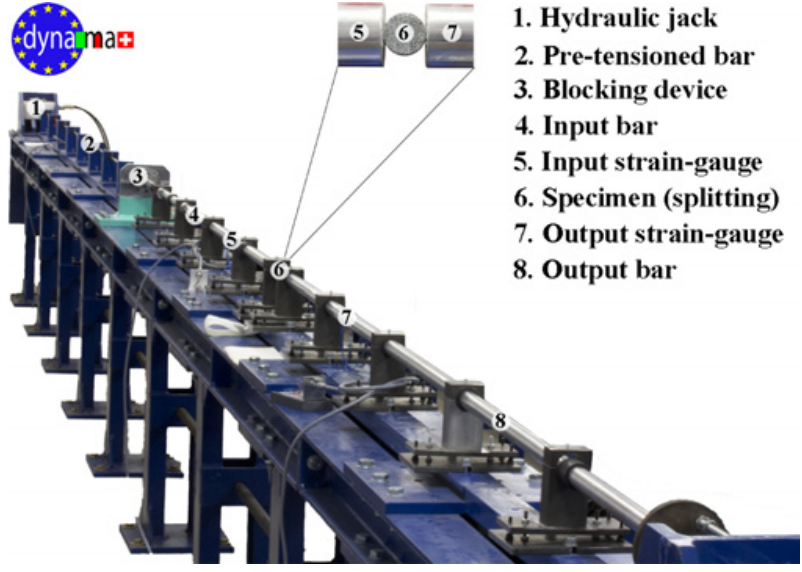

Figure 1. Modified Hopkinson bar setup for splitting tests.

a diameter of $20 \mathrm{~mm}$. Each cylinder used for direct tensile test was also notched (with a notch depth of $1.5 \mathrm{~mm}$ ). At least three specimens for each loading condition were considered.

A preliminary static characterization was initially conducted on the investigated specimens by means of a universal electro-mechanic machine with a constant strainrate of $10^{-5} \mathrm{~s}^{-1}$.

Dynamic tensile failure tests were conducted, in the DynaMat laboratory of the University of Applied Sciences of Southern Switzerland, on all the specimens, investigating medium strain-rate and the high strain-rate behaviour. The former, commonly considered as ranging from $10^{-1}$ to $10 \mathrm{~s}^{-1}$, can be induced by severe seismic actions, while the latter, varying between 10 and $10^{2} \mathrm{~s}^{-1}$, can be due to impact or blast loading. Two different experimental setups were used. For medium strain-rate tests, a hydro-pneumatic machine (HPM) was employed, whereas two modified Hopkinson bar apparatus, in tension $(\mathrm{MHBt})$ and compression $(\mathrm{MHBc})$, were used to conduct high strain-rate tensile and splitting tests, respectively.

The functioning of the hydro-pneumatic machine and the modified Hopkinson bar setup in tension have been widely reported $[14,15]$, while the modified Hopkinson bar in compression, used for splitting tests (Fig. 1), consists of a hydraulic actuator (1) that put in tension a preloading bar (2) thanks to a blocking ring (3) placed at the extremity of this bar. The preloading bar, made of high strength steel, is $6 \mathrm{~m}$ long and $12 \mathrm{~mm}$ in diameter. This bar is connected to an aluminium bar with a diameter of $30 \mathrm{~mm}$ and a length of $3 \mathrm{~m}$, working as a input bar (4)). The specimen (5) is sandwiched between the input bar and another identical bar used as output bar (6). By pulling the pre-loading bar, it is possible to drive the test by the energy stored in it. The test starts when a fragile bolt positioned between the pre-stressed bar and the hydraulic actuator (1) by suddenly breaks. Consequently, a rectangular stress wave pulse is generated and propagates through the input bar, the specimen and the output bar. By the strain gauges placed on the input and output bars, the test signals are obtained.

Each dynamic test was filmed by means of a fast recording camera MotionPro Y4-S3. 


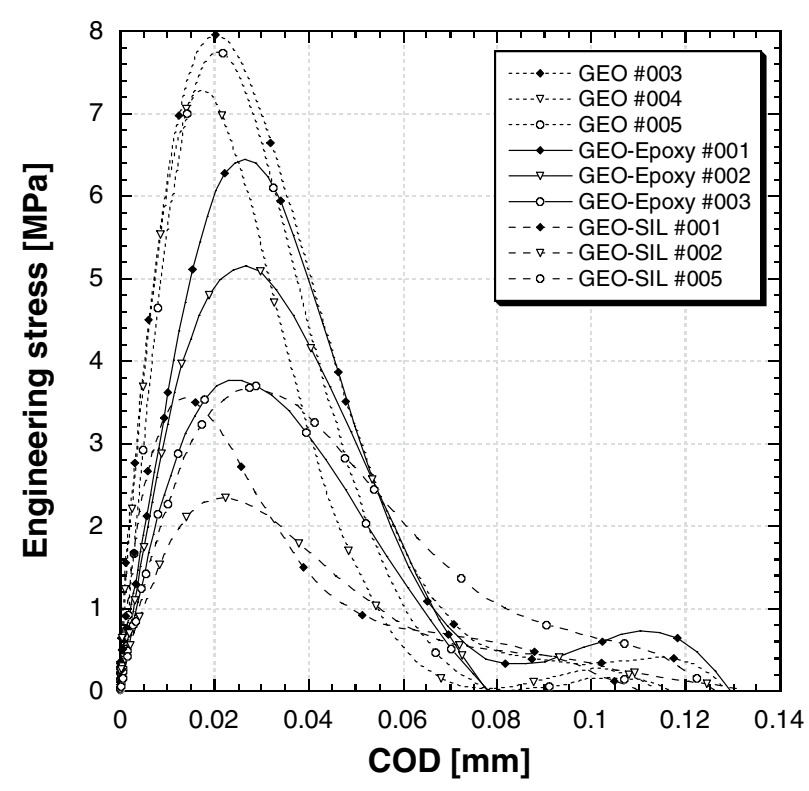

Figure 2. Tensile tests at $150 \mathrm{~s}^{-1}$.

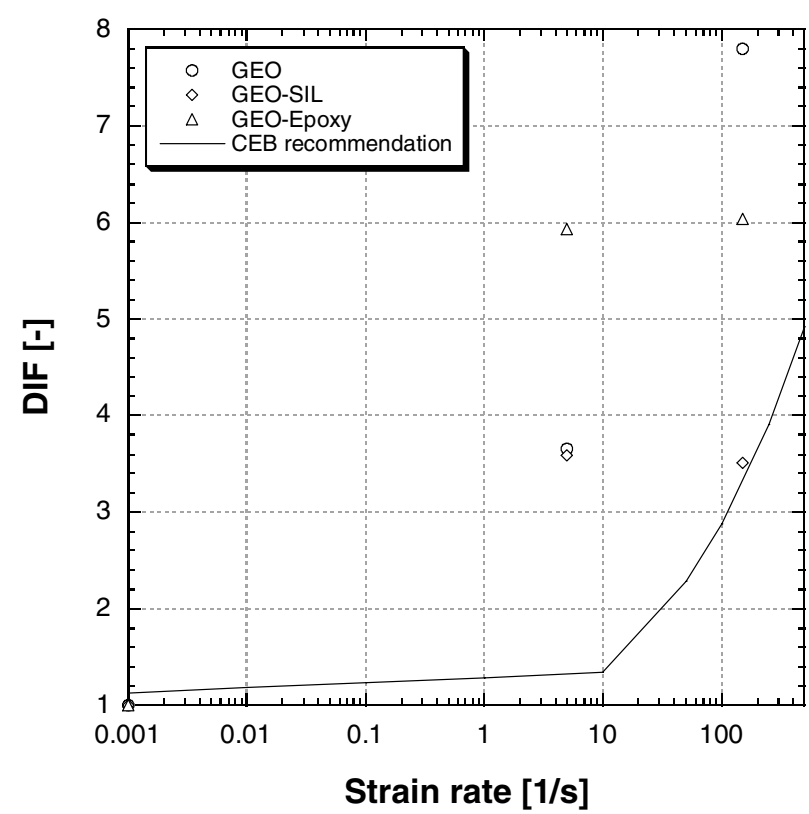

Figure 3. DIF versus strain rate.

The results are processed in terms engineering stress versus crack opening displacement (COD) and strength Dynamic Increase Factor (DIF) at different strain-rate levels.

\section{Results and discussion}

The strain rate behaviour of the three different geopolymerbased materials is hereafter discussed.

The results of the high strain rate direct tensile tests, in terms of engineering stress versus crack opening displacement, are depicted in Fig. 2. The obtained strain rate, measured in correspondence of the maximum tensile strength, is approximately $150 \mathrm{~s}^{-1}$. Is it possible to observe that the addition of epoxy and siloxanic resins led to a decrease of the maximum tensile strength with respect to
Table 1. DIF experimental parameters.

\begin{tabular}{|c|c|c|c|}
\hline & \multicolumn{2}{|c|}{$\begin{array}{c}\text { Tensile } \\
\text { tests }\end{array}$} & $\begin{array}{c}\text { Splitting } \\
\text { tests }\end{array}$ \\
\hline & $\mathbf{5 ~ s}^{-1}$ & $\mathbf{1 5 0 ~ s}^{-1}$ & $\mathbf{1 5 0 ~ s}^{-1}$ \\
\hline GEO & 3.66 & 7.80 & 3.99 \\
\hline GEO-SIL & 3.59 & 3.51 & 4.26 \\
\hline GEO-Epoxy & 5.93 & 6.04 & 3.35 \\
\hline
\end{tabular}

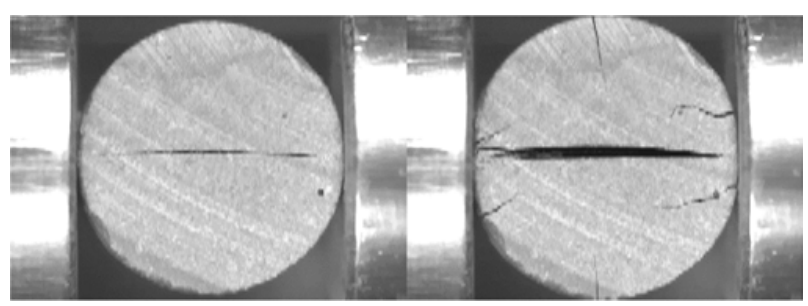

Figure 4. Pictures of a splitting test (GEO) at 1/27100 fps.

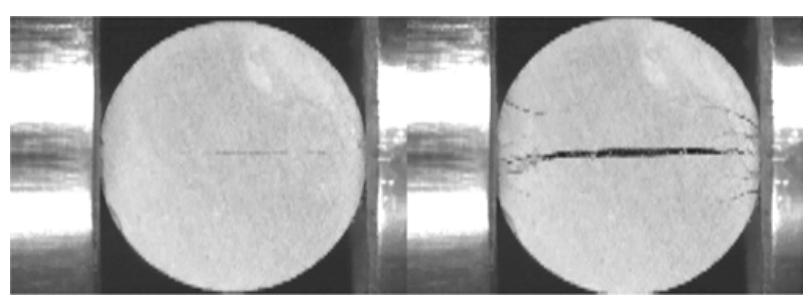

Figure 5. Pictures of a splitting test (GEO-SIL) at 1/28300 fps.

the metakaolin based geopolymer. This could be due to the different microstructures characterizing the different investigated materials $[8,13]$. In particular, GEO samples have a rather irregular microstructure with diffused nanopores due to the evaporation of the reaction water. On the contrary, the epoxy resin in the GEO-Epoxy samples consolidates within the geopolymer matrix as discrete micrometric spheres (i.e. a heterogeneous composite microstructure), affecting the overall dynamic behaviour due to their viscoelastic characteristics. GEO-SIL samples are characterized by a very compact and interconnected microstructure up to the nanometric scale that reduces the strain rate effect also for high levels of strain rate.

The strain rate behaviour can be also represented by means of the ratio between the dynamic and the static strength, commonly known as the Dynamic Increase Factor (DIF). The results in terms of DIF for both direct tensile and splitting tests have been collected in Table 1 and represented in Fig. 3. A comparison between the CEB [16] recommendation, widely used to predict the tensile behaviour of concrete under high strain rate, was also depicted in Fig. 3. It can be observed that GEO samples exhibited a remarkable change of DIF passing from the medium strain-rate and the high strain-rate level, whereas GEO-SIL and GEO-Epoxy seemed to not be affected by the two different ranges of strain rate.

The fracture development was filmed by means of a fast recording camera, highlighting different fracture evolutions: a single macro fracture was observed for both GEO (Fig. 4) and GEO-SIL (Fig. 5) materials, while a multi fracture was observed for GEO-Epoxy 


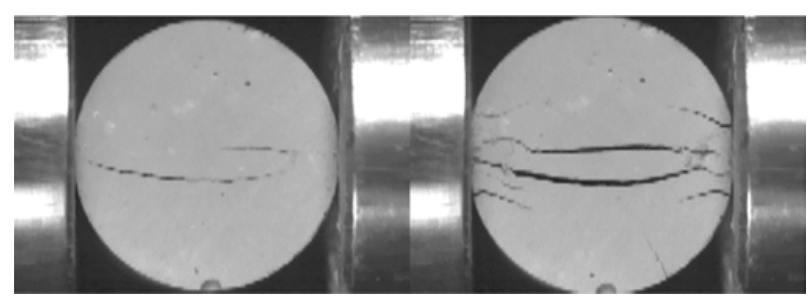

Figure 6. Pictures of a splitting test (GEO-Epoxy) at 1/28300 fps.

(Fig. 6) material. This could be due to the composite heterogeneous microstructure characterizing GEO-Epoxy samples. Indeed, different crack initiation sites could form under splitting tension loading, especially at the interface region between the epoxy micrometric spheres and the geopolymer surrounding matrix. The recorded DIF under splitting tensile loading was approximately 4 for all the three investigated materials.

\section{Conclusions}

The tensile behaviour of a metakaolin based geopolymer and two hybrid geopolymer composites was investigated at medium and high strain rates by means of dynamic tensile and splitting tests. The two types of tensile characterization tests pointed out the importance of the microstructure characterizing geopolymer-based materials with regards to strain rate effect.

Neat geopolymer material was strongly affected by strain rate (both medium and high levels), showing a remarkable shifting of the engineering stress versus crack opening displacement curves. The splitting dynamic test drew the attention also to the fracture development under high strain rate, resulting in multiple fracture sites only for GEO-Epoxy samples.

The dynamic behaviour of such geopolymer-based materials combined with aggregates for concrete products is currently under investigation by the authors.

\section{References}

[1] J. Davidovits, Geopolymer Chemistry and Applications, Institut Géopolymère 3rd ed., Saint Quentin, France (2014) www. geopolymer .org.

[2] J. Davidovits, J Therm Anal Calorim 35(2), 429-441 (1989)

[3] P. Duxson, A. Fernández-Jiménez, J. L. Provis, G. C. Lukey, A. Palomo, J. S. J. van Deventer, J Mater Sci 42(9), 2917-2933 (2007)

[4] S. Andini, R. Cioffi, F. Colangelo, C. Ferone, F. Montagnaro, L. Santoro, Adv Sci Technol 69, 123-128 (2010)

[5] A. Buchwald, M. Hohmann, K. Posern, E. Brendler, Appl Clay Sci 3, 300-304 (2009)

[6] B. Molino, A. De Vincenzo, C. Ferone, F. Messina, F. Colangelo, R. Cioffi, Materials 7, 5603-5616 (2014)

[7] C. Ferone, G. Roviello, F. Colangelo, R. Cioffi, O. Tarallo, Appl Clay Sci 73, 42-50 (2013)

[8] G. Roviello, L. Ricciotti, C. Ferone, F. Colangelo, R. Cioffi, O. Tarallo, Materials 6, 3943-3962 (2013)

[9] Z. Pan, K.N. Feng, K. Gong, B. Zou, A.H. Korayem, J. Sanjayan, J Mater Sci 48(8), 3128-3137 (2013)

[10] M. Khandelwal, P.G. Ranjith, Z. Pan, J.G. Sanjayan, Arab J Geosci 6(7), 2383-2389 (2011)

[11] K.N. Feng, D. Ruan, Z. Pan, F. Collins, Y. Bai, C.M. Wang et al., Mater Struct 48, 671-681 (2015)

[12] C. Ferone, F. Colangelo, G. Roviello, R. Cioffi, C. Menna, D. Asprone, A. Balsamo, A. Prota, G. Manfredi, Materials 6, 1920-1939 (2013)

[13] G. Roviello, C. Menna, O. Tarallo, L. Ricciotti, C. Ferone, F. Colangelo, D. Asprone, R di Maggio, E. Cappelletto, A. Prota, R. Cioffi, sumbitted to Cement and Concrete Research (to be published)

[14] E. Cadoni, Rock Mech Rock Eng, 43, 667-676 (2010)

[15] E. Cadoni, M. Dotta, D. Forni, P. Spätig, J. Nucl. Mat. 414(3), 360-366 (2011)

[16] FIB-International Federation for Structural Concrete, Model Code 2010 (2012) 